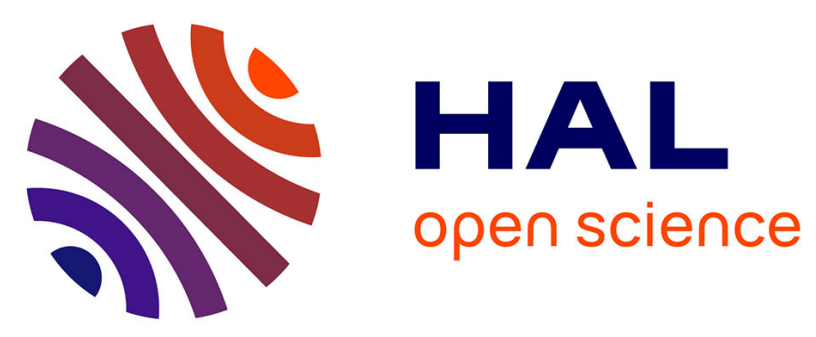

\title{
Development of a Methodology for the Analysis and Evaluation of Alternative Actions in Disruption Management in Production
}

\author{
Ben Luetkehoff, Volker Stich, Moritz Schroeter, Felix Steinlein
}

\section{To cite this version:}

Ben Luetkehoff, Volker Stich, Moritz Schroeter, Felix Steinlein. Development of a Methodology for the Analysis and Evaluation of Alternative Actions in Disruption Management in Production. 20th Working Conference on Virtual Enterprises (PRO-VE), Sep 2019, Turin, Italy. pp.143-150, 10.1007/9783-030-28464-0_13 . hal-02478759

HAL Id: hal-02478759

https://hal.inria.fr/hal-02478759

Submitted on 14 Feb 2020

HAL is a multi-disciplinary open access archive for the deposit and dissemination of scientific research documents, whether they are published or not. The documents may come from teaching and research institutions in France or abroad, or from public or private research centers.
L'archive ouverte pluridisciplinaire HAL, est destinée au dépôt et à la diffusion de documents scientifiques de niveau recherche, publiés ou non, émanant des établissements d'enseignement et de recherche français ou étrangers, des laboratoires publics ou privés. 


\title{
Development of a Methodology for the Analysis and Evaluation of Alternative Actions in Disruption Management in Production
}

\author{
Ben Luetkehoff, Volker Stich, Moritz Schroeter and Felix Steinlein \\ FIR e.V., Campus-Boulevard 55, 52074 Aachen, Germany \\ ben.luetkehoff@fir.rwth-aachen.de
}

\begin{abstract}
Due to Digital Transformation, also called Industry 4.0 or the Industrial Internet of Things, the barrier for implementing data collecting technology on the shop floor has decreased dramatically in the past years leading to an increasingly growing amount of data from a multitude of IT systems in production companies worldwide. Despite that, the production controller still relies heavily on intrinsic knowledge and intuition for the management of disruptions in production. Thanks to advances in the fields of production control and artificial intelligence, potentials for the collected data for disruption management arise. However, in order to transform data into usable information and allow drawing conclusions for disruption management in production, the relevant data-objects, disturbances and alternative actions must be known. Thus, the decision-making can be supported, reducing the decision latency and increasing benefit of alternative actions. Therefore, the goal of this paper is to discuss the prerequisites necessary to perform a data based disruption management and the methodology itself, serving as an approach to allow companies to build a data basis, classify disruptions and alternative actions in order to improve decision making in the future.
\end{abstract}

Keywords: Decision making, decision support, disruption management, reaction strategy.

\section{Introduction}

Offering customer specific products defines small and medium enterprises (SMEs), especially mechanical and plant engineering companies. This, combined with increasing product varieties and deceasing product lifecycles, leads to highly complex production processes, which in turn lead to an increasing amount of potential disturbances [1,2]. These company-internal or -external disturbances are to blame for disruptions and their effect on, for example, delivery dates. Efficient Production Planning and Control systems are the most important means to react to this changing environment [3]. However, while solutions - even automated ones - exist in the field of detailed planning, the production controller is usually left unsupported in many areas when it comes to disruption management [4]. This makes the job of a production controller increasingly difficult. They need to comprehend the effect of the disturbances 
on production as well as the outcomes of the possible alternatives with regard to the production system and the logistical target system of the company. Therefore, the goal of this paper is to introduce a methodology that supports the production controller by providing possible alternative actions in disruption management for production. The methodology is part of the research project "iProd" with the goal of developing a collaborative platform [5, 6].

This paper starts by discussing the motivation of the subject in a more detailed manner. Afterwards, the state of the art discusses the current solutions found in practice and in research. As a basis for the methodology to be developed, requirements are collected and discussed. Chapter 5 will introduce the methodology itself, covering the aspects of the data model, setting up rules as well as the analysis and derivation of alternative actions. The findings will be concluded and a short outlook on upcoming research will be given.

\section{Motivation}

Production Planning and Control plans the current production program at regular intervals in advance according to type and quantity over several planning periods. Its goal is to implement the program with given or planned capacities as economically as possible while taking unavoidable disruptions such as personnel losses, delivery delays or rejects into account [7].

Disruption management therefore is part of the short-term production management and closes the gap between regular production control and closed-loop production control [6]. The goal is a short, medium and long-term reduction of disruptions. Generally, preventive disruption management takes place to avoid disruptions before they arise, whereas reactive disruption management starts only after the occurrence of a malfunction in order to reduce its effect [8]. For this, appropriate reaction strategies (i.e. prefabricated decisions that intervene through the system and define measures to eliminate the disruption) are required. These are control processes that eliminate operational interruptions or occur as preventive measures to prevent malfunctions [9]. Dealing with disruptions in an organized way has several advantages, since the time it takes to react to a disturbance can be shortened noticeably. This leads - aside from the time-benefit - to a higher benefit of the adaption itself [10].

Within the research project "iProd", a collaborative platform is developed, that allows the analysis of production data using Artificial Intelligence (AI). The presented method is part of the project and deals with the disruption management as part of the platform. Due to the connectivity to the shop floor and IT-systems within the company, the platform notices the disruption, analyzes it and feeds it back to the system. Thus, the presented methodology is the backbone of the collaborative platform which in turn allows a closed-loop production control [6]. 


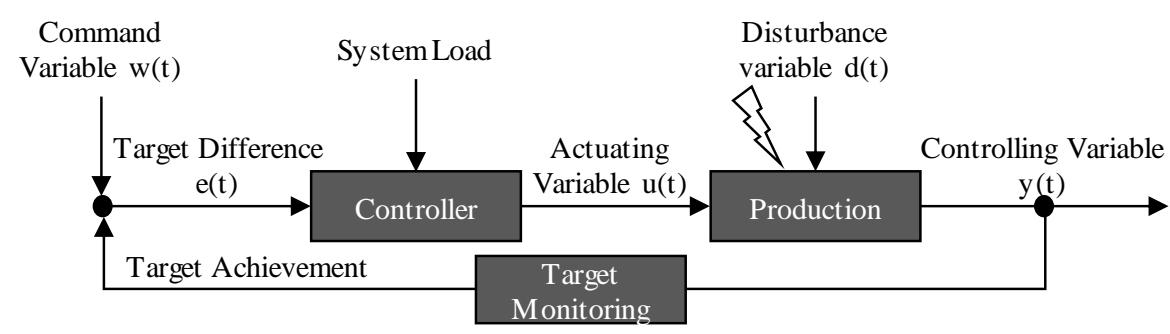

Fig. 1. Closed-loop production control [according to 11]

\section{State of the Art}

The state of the art shows an analysis of the research already available in the areas of disruption management, reaction strategies and simulation. Moreover, the industry also provides solutions in the field of disruption management, which this chapter will also discuss.

\subsection{Literature in the Field of Disruption Management, Reaction Strategies and in the Field of Simulation}

The literature review showed research focusing on the classification of disruptions in production processes as well as preventive and reactive disruption management, e.g. for customer specific production $[8,12]$. Other researchers focus on the evaluation of countermeasures. The focus here lies on mathematical models [13] or very specific areas in production, such as the assembly [14]. Moreover, there are different approaches in the field of simulation (discrete event as well as agent based) in the context of production [15-19]. Comparing the relevant literature references shows that researchbased approaches to simulation-based decision support are often limited to certain use cases or cannot be transferred to other industries.

\subsection{State of the Art in the Industry}

In practice, approaches for decision support can already be found in the area of ERP (Enterprise Resource Planning), APS (Advanced Planning and Scheduling) as well as MES (Manufacturing Execution System) systems.

ME systems integrate information relevant to production (personnel, material resources, production equipment including tools and fixtures) and link them to the planning framework conditions from the higher-level systems [20]. This way, MES generate more realistic plans than ERP systems and can react promptly to changes (e.g. due to disruptions in the production area) and calculate and initiate plan changes. ERP and MES cannot replace the production planner during planning and should rather 
support him interactively. However, the support of the production planner is prevented by the lack of transparency of the algorithms and the IT system structures, since the comprehensibility of the calculation by the production planner is a prerequisite for checking and supplementing the calculated proposal [21].

APS systems also obtain their data from ongoing value-added processes from transaction-based ERP and ME systems but deliver more exact planning results. APS systems generate plans based on advanced mathematical models. On the operational level, the detailed planning takes place throughout the entire supply chain. Here, most of the parameters and dependencies are already known; it is simply a matter of getting the optimum planning results out of the input. Thus, Companies can plan their requirements, quantities, due-dates, capacities to the point of distribution and logistics across the entire supply chain. Unfortunately, the planning horizon of APS systems ranges from days to weeks and is therefore not fast enough for short-term disruption management. The exact scope of APS systems remain to be defined.

In summary, it can be stated that there is no holistic recording and evaluation model of alternative actions for short-term disruption management. In the literature, there are various papers on aspects such as disruption management, reaction strategies, simulation and quantitative evaluation alternative actions. The research deficit is therefore a decision support system for short-term production control in the field of disruption management.

\section{Requirements}

The proposed concept must meet specific requirements to fulfill the need of the methodology. First, the data basis must be up to date and at the same time have access to historical values and planning data. Since the response time in short term production management is highly important, the evaluation of alternative actions must happen in or close to real-time. The goal of this methodology is that it either serves as an extension of existing IT systems or the production controllers themselves can work with the solution (e.g. for mobile use). Therefore, the interface must be open and allow displaying the results in a self-explanatory and user-centric manner [22].

Aside from the above-mentioned requirements, the following questions arise and need to be addressed when implementing the model: 1) Which decision cases occur in production? Which data is therefore required as input for a decision tool? 2) What effects do the potential measures have and how can these effects be quantified? 3) Which information must be prepared for the decision maker and how?

\section{Methodology}

The methodology consists of several steps that allow an evaluation of the reaction strategy and the selection of the most promising one. The first step consists of setting up the relevant data model (i.e. which data can be found where). The second step is to collect the existing and potential disruptions as well as counter measures manually (e.g. by interviewing the production controller) and automatically (e.g. by analyzing the 
historical data). Based on this, rules for the identification of future disruptions and the derivation of alternative actions. The basic framework for the methodology is illustrated in figure 2 .

\subsection{Identification of Relevant Data}

In many companies, the worker or production controller notices disruptions, however, the goal is that the system can detect them automatically. The latter is done via an automatic transfer of order data from the ERP and ME system as well as from production or machine data acquisition (PDA/MDA). Manual input from the worker is required if the data cannot be transferred from the IT systems automatically. This input may contain additional parameters for the in-house production such as shift plans (e.g. from a human resource system), amount and qualification of workers, disruptions or maintenance at resources, blocked parts as well as the availability of resources from Production Data Acquisition (PDA).

In this first step, it is necessary to define the scope of disruption management within the company in order to identify the relevant data objects. The typology of the company in focus will heavily determine the needed data. For example, while for one company the batch-number and size may be relevant, others simply do not produce batches. This data then needs to be allocated (i.e. which databank stores this data, e.g. ERP system) and its meaning defined. This means that, even within the same company, the understanding of the used vocabulary may differ.

\subsection{Collecting Disruptions, Alternative Actions and Rules}

The goal of the digital transformation in production is to support the worker and to turn their implicit knowledge into usable data for IT systems. Thus, when setting up this methodology for disruption management, the worker needs to be interviewed and the known disruptions as well as their countermeasures need to be collected. If the company already has a reliable database, this can also be used in order to identify disruptions and alternative actions from the past. This step usually results in unveiling missing data which then needs to be added to the data model.

In the presented project, a matrix has been developed, that groups the disruptions in reference objects. Possible causes, their effect and characteristic define the reference object in more detail. The alternative actions are grouped in reaction strategies (i.e. delay, delegation, replanning, relaxation, negotiation and cancellation). Based on the matrix, the reaction strategies can be allocated to the disruptions and thus alternative actions be derived.

\subsection{Analyze the Data and Knowledge Base}

When a disruption occurs, the first step is to identify the disruption. Depending on the grade of automation within the company, the reaction for disruptions with low impact 
can be performed automatically, without requiring an intervention of an employee. At the same time, there are disruptions that can solely be solved by manual interventions from an employee. The type of disruption that is the focus of this paper is the type that can be encountered semi-automatically. Here, part of the input can come from the system and the other part still requires some kind of manual input. [23]

After the disruption has been identified, it needs to be analyzed in more detail. This is necessary in order to see what the reaction strategy and alternative actions looked like in the past. The AI-tool (Jupyter Notebook) is used to extract the necessary data and analyze them separately regarding the disruption. Afterwards, the analyzed data is fed back via the interface ${ }^{1}$. The result of this analysis depends on the defined scope. An example is a clustering of disruptions according to their conditions (e.g. product variant or past alternative actions) in order to anticipate further disruptions, and therefore enables a short-, medium- and long-term disruption management.

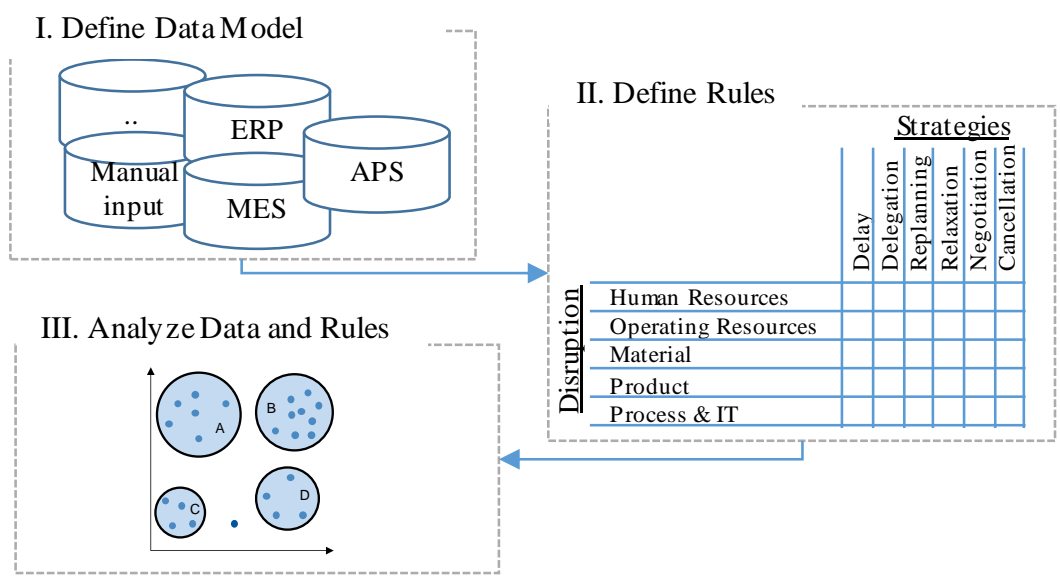

Fig. 2. Methodology to approach disruptions

\section{Conclusion and Outlook}

Companies try to encounter the turbulent market, customer specific production and thus higher susceptibility to disruptions in production by collecting data and supporting the worker with IT systems. While many aspects of production have already been facilitated by this digital transformation, the disturbance management remains unsupported and mostly based on implicit knowledge and intuition of the worker. In order to improve the decision making process in disruption management, this paper discussed a possible methodology by discussing the data model, rules (disruptions and alternative actions) as well as their analysis briefly. The findings can be used as an extension of existing IT systems or for standalone support systems. However, within the presented project, the methodology still involves many manual steps and is not yet

1 In the presented project, a platform is developed that uses REST API interfaces in order to feed data back to the IT systems or to a web-based dashboard. For more information please see www.projekt-iprod.de and $[6,5]$. 
ready for a direct implementation. Future research therefore needs to focus the area of data models for disruption management as well as their ontology. Moreover, the methodology needs validation in practice and standardization of the analysis using AI. For companies it is crucial to collect the implicit knowledge of the worker in order to allow the described methodology in practice.

\section{Acknowledgement}

The European Regional Development Fund (ERDF) funded the presented research (grant number EFRE/ERDF-0800924). The authors would like to thank the European Regional Development Fund for the kind support and making this research possible.

\section{References}

1. ElMaraghy, H., Schuh, G., ElMaraghy, W. et al. (2013): Product variety management. CIRP Annals 62(2): 629-652

2. Ferreira, F., Faria, J., Azevedo, A. et al. (2017): Product lifecycle management in knowledge intensive collaborative environments: An application to automotive industry. International Journal of Information Management 37(1): 1474-1487

3. Böckenkamp, A., Mertens, C., Prasse, C. et al.: A Versatile and Scalable Production Planning and Control System for Small Batch Series. In: Jaschke S, Brecher C, Song $\mathrm{H}$ et al. (eds) Industrial internet of things: Cybermanufacturing systems / Sabina Jaschke, Christian Brecher, Houbing Song, Danda B. Rawat, editors. Springer, Cham, Switzerland, pp 541-559 (2017)

4. Schuh G, Potente T, Thomas C et al. (eds): Cyber-Physical Production Management: Advances in Production Management Systems. Sustainable Production and Service Supply Chains. Springer Berlin Heidelberg (2013)

5. Luetkehoff, B., Blum, M., Schroeter, M.: Self-learning Production Control Using Algorithms of Artificial Intelligence. In: Camarinha-Matos LM, Afsarmanesh H, Fornasiero R (eds) Collaboration in a Data-Rich World, vol 506. Springer International Publishing, Cham, pp 299-306 (2017)

6. Luetkehoff, B., Blum, M., Schroeter, M.: Development of a Collaborative Platform for Closed Loop Production Control. In: Camarinha-Matos LM, Afsarmanesh H, Rezgui Y (eds) Collaborative Networks of Cognitive Systems. Springer International Publishing, Cham, pp 278-285 (2018)

7. Wiendahl, H.-P.: Betriebsorganisation für Ingenieure, 8., überarb. Aufl. Hanser, München (2014)

8. Schwartz, F., Voß, S.: Störungsmanagement in der ProduktionSimulationsstudien für ein hybrides Fließfertigungssystem. Zeitschrift für Planung \& Unternehmenssteuerung 15(4): 427-447 (2004)

9. Opfermann, K.: Kostenoptimale Zuverlässigkeit produktiver Systeme. Dr. Gabler (1968) 
10. Gu, X., Jin, X., Ni, J. et al. (2015): Manufacturing System Design for Resilience. Procedia CIRP 36: 135-140

11. Wiendahl, H.-P.: Fertigungsregelung: Logistische Beherrschung von Fertigungsabläufen auf Basis des Trichtermodells. Hanser, München (1997)

12. Wünscher, T.: Störungsmanagement im Entwicklungs-und Herstellungsprozess komplexer, kundenindividueller Produkte. Cuvillier (2010)

13. Stich V, Schröter M, Jordan F et al. (eds): Assessment of Counter-Measures for Disturbance Management in Manufacturing Environments. Springer (2017)

14. Burggräf, P., Wagner, J., Lück, K. et al.: Cost-benefit analysis for disruption prevention in low-volume assembly. Production Engineering 11(3): 331-342 (2017)

15. Melouk, S., Fontem, B., Waymire, E. et al. (2014): Stochastic resource allocation using a predictor-based heuristic for optimization via simulation. Computers \& Operations Research 46: 38-48

16. Krenczyk, D., Olender, M. (2015): Simulation Aided Production Planning and Scheduling Using Game Theory Approach. AMM 809-810: 1450-1455

17. Burggraef, P., Wagner, J., Dannapfel, M. et al.: Simulating the benefit of disruption prevention in assembly. Journal of Modelling in Management (2018)

18. Greenwood, A., Vanguri, S., Eksioglu, B. et al.: Simulation Optimization Decision Support System for Ship Panel Shop Operations. In: Proceedings of the Winter Simulation Conference, 2005. IEEE, pp 2078-2086 (2005)

19. Galaske, N., Anderl, R. (2016): Disruption Management for Resilient Processes in Cyber-physical Production Systems. Procedia CIRP 50: 442-447

20. Kletti, J.: MES - Manufacturing Execution System: Moderne Informationstechnologie Zur Prozessfähigkeit Der Wertschöpfung. Springer, Dordrecht (2006)

21. Alexandre Gachet, Pius Haettenschwiler (2003): A Decentralized Approach to Distributed Decision Support Systems. Journal of Decision Systems 12(2): 141158

22. Nyhuis, P., Mayer, J., Kuprat, T.: Die Bedeutung von Industrie 4.0 als Enabler für logistische Modelle. Industrie 4: 79-100 (2014)

23. Meissner, J.: Adaptives Abweichungsmanagement in der Fertigungssteuerung bei Kleinserien. Dissertation, RWTH-Aachen (2018) 